Review Paper

\title{
Dual Specificity of Alternaria to Cause Plant Disease and Allergic Reactions
}

\author{
Md Ehsanul Haque ${ }^{1 *}$, Most Shanaj Parvin ${ }^{2,3}$
}

\author{
${ }^{1}$ University of North Dakota, Grand Forks 58203, USA \\ ${ }^{2}$ Bangladesh Agricultural Research Institute, Joydebpur, Gazipur-1701, Bangladesh \\ ${ }^{3}$ Gottfried Wilhelm Leibniz Universität Hannover, Germany
}

*Corresponding Author: Email: mdehsanul.haque@ndus.edu

Received 20 December 2020; Accepted 02 February 2021

\begin{abstract}
Alternaria is a ubiquitous fungal genus which includes endophitic, saprophytic and pathogenic species. They can cause pre and post-harvest diseases in numerous crops and world-wide economic losses. Alternaria spp. with small conidia size, less than $60 \mu \mathrm{m}$, are categorized as small spore Alternaria. Alternaria alternata, Alternaria arborescens, and Alternaria tenuissima are among the most commonly reported small spore Alternaria spp. These species represent a disease complex on some hosts, often isolated from a single plant. Among the species, there remain overlapping morphological characteristics, and often it is not easy to differentiate the species microscopically. These pathogens usually appeared in anamorph, or asexual reproductive stage and the sexual reproduction is mostly known. There are very few studies on the genetic structure and evolutionary trajectory of the pathogen populations in the agroecosystems. The conidia are usually produced on leaf lesions, and often colonize in wounds. Asexual spores are larger than other anemophilous pollen and fungal spores, but they are found in high quantities in the air. The spores can disperse through air currents and rainfall. However, they are also known to be an allergenic fungus. A recent study in Europe has shown that Alternaria sp and its aerial asexual spores' inhalation of patients escalated the allergy by $8.9 \%$. And it has also been pronounced as a risk factor for the development, persistence, and severity of asthma. Hence, it is necessary to identify the indoor and outdoor Alternaria species that are particularly involved in allergenic or asthma reactions.
\end{abstract}

Keywords: Alternaria, Fungi, Diseases, Asthma

\section{INTRODUCTION}

Alternaria, is a ubiquitous fungus that can sustain under a full range temperature. This fungus is predominantly saprophytic microorganisms or plant pathogenic (Simmons 2007). The taxonomy is based primarily on the morphology and the development of conidia and conidiophores. Secondly, the host plant association and colony morphology. The Alternaria conidiophores pale brown, simple or branched, bearing catenulate conidia at the apex and fertile apical parts. The typical structure of the conidia is often long chained ( 9 or more), acropetally developed, polymorphous, transverse (1-9) and several longitudinal or oblique septate (Konstantinova et al. 2002). There is a profound variability in conidial shape and size. However, Alternaria conidia are believed to be a well-known biological contaminant and a source of outdoor aeroallergen, and it is found to be associated with asthma. In 2006, National Institute Health reported species; Alternaria alternate as a critical risk factor for asthma in indoor environments. Alternaria conidia were collected from dust samples in kitchen floors, living room sofa, and chairs, and also from the bedroom concentration and later it was measured via polyclonal antigen-antibody assay. Exposure to Alternaria antigen found to increase the active asthma symptoms (Salo et al. 2006). Recent studies have shown that Alternaria and other allergenic fungi can colonize in indoor environments, and it can elevate the fungal aeroallergen exposure level (Gabriel et al. 2016). Notably, Alternaria genus has a large number of recognized species, and they have overlapping morphological characteristics. Hence the identification of true species groups involved in allergenic reactions is somehow challenging. Apart from that, there is a diverse spore dimension range, and it overlaps among the species. Conidial morphology and catenation are affected by environmental factors; for instance; light and humidity. Therefore, the morphological characterization may be unreliable (Kgatle et al. 2018; Nishikawa and Nakashima 2013). Over the last two decades, simple sequence repeats or microsatellite markers have been used for population genetics studies that enriched our understanding of the fungal biology of plant pathogens. Nevertheless, 
it has complicacy in mutation-migration-drift equilibrium, null alleles, homoplasy, and genome-wide patterns of diversity (Biasi et al. 2016; Vaghefi et al. 2017).

On the other hand, single nucleotide polymorphism (SNPs) offers genome-wide coverage, but it requires a higher number of loci to be analyzed. These criteria hindered the use of SNPs in population genetics studies of non-model organisms (Garvin et al. 2010; Vaghefi et al. 2017). However, genotyping by sequencing (GBS) method involves discovering single nucleotide polymorphisms (SNP) to facilitate genome-wide association studies (GWAS) and reduce genome complexity. After digestion with restriction enzymes, PCR is followed up to increase fragments pool, and GBS libraries are sequenced using next-generation sequencing technologies. It better reflects the multitude of polymorphic markers that help to understand the genome-wide genetic diversity of populations and improve resolution to identify fine-scale genetic variation or detect rare recombination events (Elshire et al. 2011). Recently, genotyping by sequencing approach confirmed the global genotype flow in Cercospora beticola populations (Elshire et al. 2011, Vaghefi et al. 2017). Therefore, we intend to review the occurrence of global genotype flow in Alternaria populations and its allergenic gene cluster via GBS approach. It can help to estimate the clonal diversity, differentiation, and structure of Alternaria populations that are particularly involved in the allergenic reaction.

\section{PLANT DISEASE ASPECTS OF ALTERNARIA}

Alternaria is known as a plant pathogenic saprophytic opportunistic pathogen and regarded as a weak filamentous fungus. They cause brown leaf spots (in citrus and sugar beet), leaf blight (in carrot and sunflower), stem canker (in tomato), seed, and root rot (in carrot) (Hou et al. 2016; James J. F. 2004; Kgatle et al. 2018; Khan et al. 2020). It has different hosts varying on its forma species, for instance; the cruciferous-vegetable family, the Solanaceae family including potato and tomato, and the grass family. Even though, it involves crop damage in the field and escalates post-harvest decay. High humidity favors plant disease, infection, and sporulation, while lower humidity conducive for conidial dissemination. The pathogen may yield to secondary infection phase if there are abundant spores or inocula available in the field, and they undergo overwintering on crop debris. The pathogenic species produces harmful mycotoxin which can contaminate plant products and are often subjected to cancer.

Alternaria leaf blight reported firstly in Germany in 1855; it's described as a sporadic disease in carrot in Northern Europe. While in the USA, it was first reported in Louisiana in 1890, the causal agent identified as Macrosporium carotae. Subsequently, it was reported in Massachusetts and Florida, and since then the damage was found to be pronounced in carrot growing areas in the other parts of the world (James J. F. 2004). Including, A. alternata and other species of Alternaria, for example; A. radicinia and A. dauci are reported to be isolated from carrot seeds and roots in all growth stages. And these groups often cause severe infection reduces carrot's root size and yields.

Similarly, Alternaria leaf blight of sunflower is considered as a significant paramount disease as it hampers up to $80 \%$ and $30 \%$ seed and oil production, respectively. Causal organism A. helianthi coined as the prime cause of Alternaria leaf spot of sunflowers. Several other Alternaria species have been found to be pathogenic to sunflower; for example; A. alternata Kiessler, A. helianthicola Simmons, A. helianthicola Rao and Rajagopalan, A. leucanthemi Nelen, A. longissimi Deighton and MacGarvey, A. protenta Simmons, A. zinnia Ellis, and A. teuissima Wiltshire. Lately, A. alternata is synonymized with A. teuissima Recently, A. alternata reported to causing leaf blight in South Africa in 2018; these findings indicated that Alternaria genus belongs to a complex phylogenetic grouping (Woudenberg et al. 2015; Kgatle et al. 2018; Nishikawa and Nakashima 2013). Disease management is a bit challenging due to the unusual timing of infection. However, well-controlled timely irrigation and regular fungicide application can be an alternative approach to minimize disease severity. Resistance development to the fungicide has become a critical issue for the management of the disease.

\section{ALLERGENICITY OF AIRBORNE ALTERNARIA SPORES}

The fungi grow both indoor and outdoor environment. Consequently, the increasing exposure level may result in profound health issue. For most people for most of the time, contact with this fungi/spores' triggers neither inflammation nor adaptive immunity. In some cases, though certain kinds of harmless molecules can stimulate a primary adaptive immune response that generates immunological memory. On subsequent exposures to this type antigens, the secondary immune response instigates inflammation and tissue damage. This unnecessary overreactions of the immune system to innocuous environmental antigen termed as hypersensitivity reactions (HR) or allergic reactions.

Some population group is more sensitive to an allergen of A. alternata. Mold sensitization, specifically to A. alternata, seems to be much more prevalent in the asthmatic population. A significant level of A. alternata allergen has been reported in farms where the occupational worker is at higher risk even it can extend to the life threated level 
(Prester and Macan 2010). A. alternata aeroallergens mediate Type I HR that involve IgE-antibody often lead mild allergic reactions. In sever case, this antigen impaired lung function and repeated encountering cause severe allergic reaction. Notably, the sensitization to A. alternata may result in deaths related to asthma. Other Research found that Alternaria allergens are a diverse group of molecules that possess different chemical and biological properties. As of now 17 IgE-inducing proteins of Alternaria have been recognized. Among them, Alt a 1 reported on the cell wall of airborne conidia (or spores). Later, this gets into the respiratory tract and elicit allergic reactions to the infected persons (Twaroch et al. 2012). Other study showed that the population sensitive to A. alternata presents approximately $80 \%$ of the sensitization prevalence by Alt a. This allergen had been reported in A. alternata and close related species (Gutierrez-Rodriguez et al. 2011). The relative abundance of Alt a 1 has been reported mostly in germinated conidia. Other research reported a homolog of Alt a 1 in A. brassicicola to cause infection in A. thaliana. The Alt a 1 was found to induce the expression of plant defense proteins that belongs to the PR5-TLP family (GomezCasado et al. 2014). This study demonstrated that Alt a 1 to be connected with pathogenesis. Other research group indicated that Alt a 1 is a conserved gene in other species of Pleosporaceae (Gabriel et al. 2016).

Other pilot study demonstrated that the allergenicity of Alternaria varies on the season, climatic conditions, source area or habitat types and species diversity (Grewling et al. 2018).

\section{THE LIFE CYCLE OF ALTERNARIA}

The life cycle begins with asexual spores or long chain conidia produced on brown leaf spots, and often colonizes in plant stems and roots; it results in black rot or wounds. It can disperse through air currents and rainfall. On the flip-side, conidia dispersal halted with prolonged high winds, long periods of leaf wetness and cold temperature. High humidity favors disease infection and sporulation, while lower humidity conducive for conidial dissemination. If the environmental condition is favorable, it paves the way of secondary infection, and the conidia spread very rapidly in the field. Temperature study at $24^{\circ} \mathrm{C}$ has observed to increasing damage with increasing hours of leaf wetness from 8 to $56 \mathrm{~h}$. Conidia and conidiophores are produced at a temperature range between 10 to $28^{\circ} \mathrm{C}$ and relative humidity from 96 to $100 \%$. Conidia became airborne and released as the relative humidity drops. Spores deposited on leaf surfaces would germinate, and infection occurs under appropriate temperature and leaf wetness conditions (Langenberg et al. 1977).

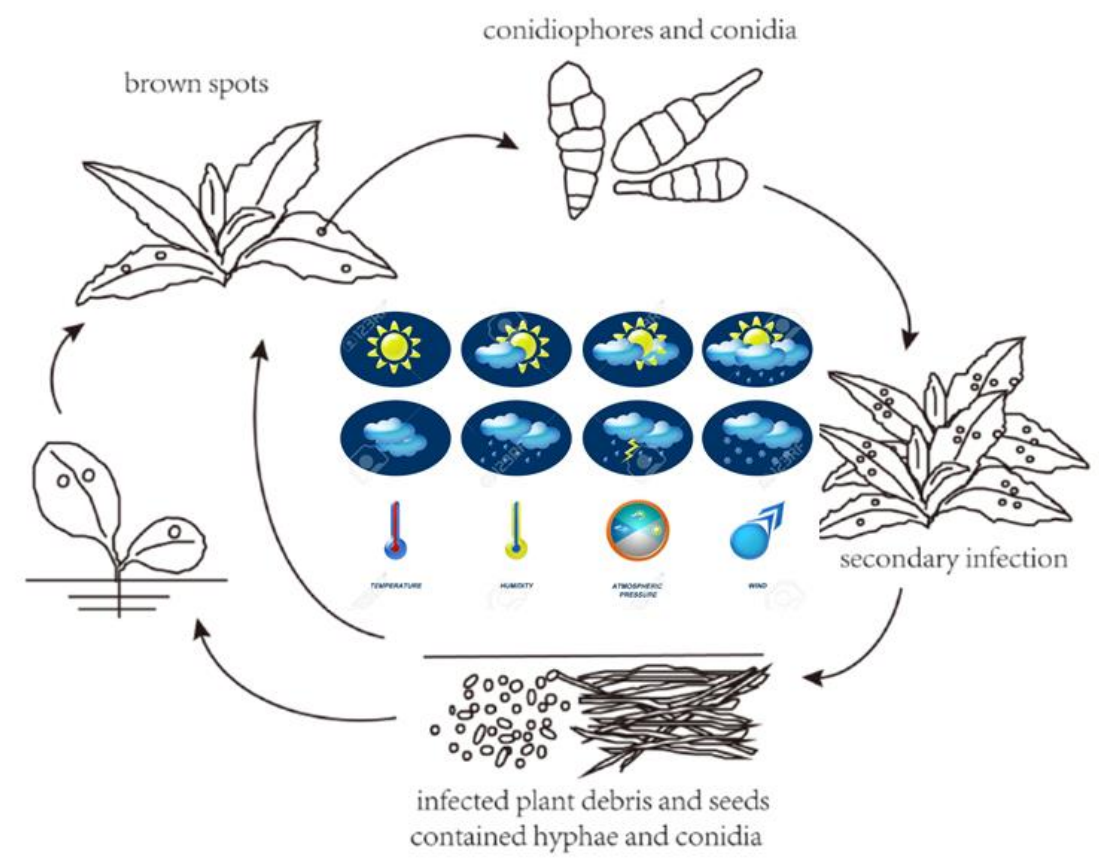

Fig. 1: Lifecycle of Alternaria (modified from Hou et al. 2016)

It causes significant loss of agriculture production, and the produced mycotoxins threaten the health and animals (Hou et al. 2016). The pathogen usually overwinters as a mycelial form in seed, crop debris, and diseased volunteer. Alternaria leaf blight reduces the photosynthetic surface area of the plants which ultimately affect the desired yields. However, a single control measure is not sufficient to control the high disease pressure. Several integrated approaches 
for instance; clean seed, crop rotation, field sanitation, resistant cultivar selection, and fungicide applications (James J. F. 2004).

\section{BIOLOGY AND GENOME OF ALTERNARIA}

The genus grows well in artificial media, for example in Potato Dextrose Agar (50\% PDA) and Water Agar. The hyphae are sub-hyaline to olive-brown and septate. The conidiophores are olive-brown and flexuous with a single terminal or one or two geniculate conidiogenous sites. Conidium is usually borne singly and appeared as long conidial chain. Conidium is medium to dark brown, long ellipsoid to obclavate, 60-100 x 15-25 $\mu \mathrm{m}$ (spore body), with 7 to 11 transepta and 1 to 3 longisepta in fewer than half of the transverse segments. Mature conidium is rostrate with a terminal filamentous beak $80-250 \times 5 \mu \mathrm{m}$ tapering distally. Teleomorph or sexual reproduction stage is mostly unknown (James J. Farrar 2004).

Lately, a draft genome sequence of A. alternata (ATCC 34957) completed by PacBio technology, and it assembled into 27 scaffolds with a total genome size of $33.5 \mathrm{Mb}$. The largest scaffolds reported up to 3.97 Mb (Hai D. T. Nguyen 2016).

\section{MOLECULAR DETECTION METHODS AND ITS IMPORTANCE}

The large species group of Alternaria have been mostly illustrated based on morphology and host-specificity, even though it is speculated to yield minimal molecular variation. Therefore, it is challenging to differentiate the Alternaria species. Moreover, synonymize certain species is often controversial. Conidial size and shapes mainly varied due to the biotic and abiotic stress-related issues. Therefore, among the species in Alternaria, there remains a morphological ambiguity. So, far multiple molecular methods tested for differentiating the small-spores Alternaria species, for example, random amplified polymorphic DNA (RAPD), amplified fragment length polymorphism (AFLP), selective subtractive hybridization, and sequence characterized amplified genomic regions. But these methods yielded with some limitation to differentiate the morphospecies described within Alternaria genus. However, for asexually reproduced Alternaria genus, the ITS region in the ribosomal RNA (rRNA) operon has been accepted as the formal fungal barcode. The internally transcribed communities and it is widely used in taxonomy and molecular phylogeny of fungi. The internal transcribed spacer (ITS) regions are the spacer DNA situated between the small-subunit ribosomal RNA (rRNA) and large subunit rRNA genes in the chromosomes. The rRNA maturation process followed by the excisions of the external transcribed sequence (5' ETS) and ITS. So far it has been used in molecular systematics; within the species and also to genus level, for instance; the universal ITS1 + ITS2 primers that allow selective amplification of fungal sequences (De Beeck et al. 2014). A recent study has conducted in the Andalusia region, Southern Spain in 2017, P. terebinthus plants observed leaf blight symptoms in a commercial nursery. Later, A. alternata, isolate ColPat-420 has been detected via sequencing the internal transcribed spacer region (ITS) and RNA polymerase second largest subunit (RPB2) with primers ITS4/ITS5 (Lopez-Moral et al. 2018).

In another study, Alternaria alternata infections that cause brown leaf blight of shenguyon in China in 2017. The molecular detection was confirmed via the internal transcribed spacer region (ITS) region of rDNA, and a histone gene was amplified using the primers ITS1 /ITS5 and H3-1a/H3-1b, respectively (Wei et al. 2018). Nevertheless, the pathogen can be detected through the ITS, but the multi-gene pathogen detection is strong enough to withstand most challenges.

Against this backdrop, genotyping-by-sequencing (GBS) approach now been profoundly promising for highly diversified and large genomic species. There are some merits to this approach, for example; it can be generalized to any species at low-cost, it can be done quickly, extremely specific, simple and highly reproducible. It can reach the essential regions of the genome that are inaccessible to sequence capture approaches. Furthermore, genomic libraries developed that contains reduced genomic complexity (Elshire et al. 2011).

This approach considers methylation-sensitive restriction enzymes (REs). Thus, repetitive regions of genomes are minimized, and it facilitates to capture low copy regions with two to three times the higher efficiency. Besides, it simplifies computational alignment problems in species with a high level of genetic diversity.

\section{CONCLUSION}

Fungi have received little attention as a potential source of Allergenic reaction compared to the common environmental sources. Previously, Alternaria has not been considered the main allergen fungus, recently the prevalence of the species has been reported as a source of airborne sensitizer allergen, and they found to grow both indoor and outdoor environment. Lately, A. alternata allergies continue to escalate, accentuating research on the 
dormant role of the fungal species and its allergenic components, particularly in asthma, makes sense. Although establishing correlations of A. alternata exposure and allergic symptoms remains problematic to determine. Apart from that Alternaria genus comprises with 300 species and they all have overlapping morphological characteristics. However, it is speculated that other species might have potential health effects. Against the backdrop, GBS may help to unveil the genomes of the Alternaria species and to pull down the allergenic gene family. Furthermore, it is paramountly important to use a multidisciplinary approach using both molecular tools and epidemiologic to accurately evaluate the exposure trends to A. alternata and its allergens and to determine the potential health effects.

Acknowledgements: We are very much thankful to Dr. Julie, NDSU for technical guidance.

Conflict of Interest: We declare no conflict of interest

\section{REFERENCES}

Biasi, A., Martin, F. N., Cacciola, S. O., Lio, G. M. D., Grunwald, N. J., Schena, L. (2016). Genetic Analysis of Phytophthora nicotianae Populations from Different Hosts Using Microsatellite Markers. Phytopathology 106:1006-1014.

De Beeck, M. O., Lievens, B., Busschaert, P., Declerck, S., Vangronsveld, J., Colpaert, J. V. (2014). Comparison and Validation of Some ITS Primer Pairs Useful for Fungal Metabarcoding Studies. Plos One 9.

Elshire, R. J., Glaubitz, J. C., Sun, Q., Poland, J. A., Kawamoto, K., Buckler, E. S., Mitchell, S. E. (2011). A Robust, Simple Genotyping-by-Sequencing (GBS) Approach for High Diversity Species. Plos One 6.

Garvin, M. R., Saitoh, K., Gharrett, A. J. (2010). Application of single nucleotide polymorphisms to non-model species: a technical review. Molecular Ecology Resources 10:915-934.

Gabriel, M. F., Postigo, I., Tomaz, C. T., Martinez, J. (2016). Alternaria alternata allergens: Markers of exposure, phylogeny and risk of fungi-induced respiratory allergy. Environment International 89-90:71-80.

Gomez-Casado, C., Murua-Garcia, A., Garrido-Arandia, M., Gonzalez-Melendi, P., Sanchez-Monge, R., Barber, D., Pacios, L. F., Diaz-Perales, A. (2014). Alt a 1 from Alternaria interacts with PR5 thaumatin-like proteins. Febs Letters 588:1501-1508.

Grewling, L., Nowak, M., Szymanska, A., Kostecki, L., Bogawski, P. (2018). Temporal variability in the allergenicity of airborne Alternaria spores. Medical mycology.

Gutierrez-Rodriguez, A., Postigo, I., Guisantes, J. A., Sunen, E., Martinez, J. (2011). Identification of allergens homologous to Alt a 1 from Stemphylium botryosum and Ulocladium botrytis. Medical Mycology 49:892896.

Hai D. T. Nguyen, a. C. T. L., a C. André Lévesque,a Tom Gräfenhanb. (2016). Draft Genome Sequence of Alternaria alternata ATCC 34957. American Society for Microbiology.

Hou, Y. J., Ma, X., Wan, W. T., Long, N., Zhang, J., Tan, Y. T., Duan, S. C., Zeng, Y., Dong, Y. (2016). Comparative Genomics of Pathogens Causing Brown Spot Disease of Tobacco: Alternaria longipes and Alternaria alternata. Plos One 11.

James J. F., B. M. P., Michael D. (2004). Alternaria Diseases of Carrot. Plant Disease 88:776-784.

James J. Farrar, B. M. P., R. Michael Davis. 2004. Alternaria Diseases of Carrot. Plant Disease 88:776-784.

Kgatle, M., Truter, M., Ramusi, T., Flett, B., Aveling, T. (2018). Alternaria alternata, the causal agent of leaf blight of sunflower in South Africa. European Journal of Plant Pathology 151:677-688.

Khan, M. F. R., Haque, M. E., Bloomquist, M., Bhuiyan, M. Z. R., Brueggeman, R., Zhong, S., Poudel, R. S., Gross, T., Hakk, P., Leng, Y., Liu, Y. (2020). First Report of Alternaria Leaf Spot Caused by Alternaria tenuissima on Sugar Beet (Beta vulgaris) in Minnesota, USA. Plant Disease 104:580-580.

Konstantinova, P., Bonants, P. J. M., van Gent-Pelzer, M. P. E., van der Zouwen, P., van den Bulk, R. (2002). Development of specific primers for detection and identification of Alternaria spp. in carrot material by PCR and comparison with blotter and plating assays. Mycological Research 106:23-33.

Langenberg, W. J., Sutton, J. C., Gillespie, T. J. (1977). Relation of weather variables and periodicities of airborne spores of alternaria-dauci. Phytopathology 67:879-883.

Lopez-Moral, A., Agusti-Brisach, C., Raya-Ortega, M. C., Roca, L. F., Lovera, M., Arquero, O., Trapero, A. (2018). First Report of Alternaria alternata Causing Leaf Blight in Pistacia terebinthus in Spain. Plant disease: PDIS02180209PDN-PDIS02180209PDN.

Nishikawa, J., Nakashima, C. (2013). Taxonomic Characterization and Experimental Host Ranges of Four Newly Recorded Species of Alternaria from Japan. Journal of Phytopathology 161:604-616. 
Prester, L., Macan, J. (2010). Determination of Alt a 1 (Alternaria alternata) in poultry farms and a sawmill using ELISA. Medical Mycology 48:298-302.

Simmons, E. G. (2007). Alternaria : an identification manual : fully illustrated and with catalogue raisonné 17962007. CBS Fungal Biodiversity Centre, Utrecht, The Netherlands.

Salo, P. M., Arbes, S. J., Jr., Sever, M., Jaramillo, R., Cohn, R. D., London, S. J., Zeldin, D. C. (2006). Exposure to Alternaria alternata in US homes is associated with asthma symptoms. Journal of Allergy and Clinical Immunology 118:892-898.

Twaroch, T. E., Arcalis, E., Sterflinger, K., Stoeger, E., Swoboda, I., Valenta, R. (2012). Predominant localization of the major Alternaria allergen Alt a 1 in the cell wall of airborne spores. Journal of Allergy and Clinical Immunology 129:1148-1149.

Vaghefi, N., Kikkert, J. R., Bolton, M. D., Hanson, L. E., Secor, G. A., Nelson, S. C., Pethybridge, S. J. (2017). Global genotype flow in Cercospora beticola populations confirmed through genotyping-by-sequencing. Plos One 12.

Wei, M., Chen, J. M., Fu, B. Z., Li, G. Y., Wang, X. S. (2018). First Report of Brown Leaf Blight of Shenguyou (Staphylea bumalda) Caused by Alternaria alternata in China. Plant Disease 102:2034-2034.

Woudenberg, J. H. C., Seidl, M. F., Groenewald, J. Z., de Vries, M., Stielow, J. B., Thomma, B. P. H. J., Crous, P. W. (2015). Alternaria section Alternaria: Species, formae speciales or pathotypes? Studies in Mycology:1-21. 